Check for updates

Cite this: RSC Adv., 2017, 7, 42549

Received 3rd April 2017

Accepted 29th August 2017

DOI: $10.1039 / c 7 r a 03805 d$

rsc.li/rsc-advances

\section{Characterizing the binding interaction between ultrafine carbon black (UFCB) and catalase: electron microscopy and spectroscopic analysis $\uparrow$}

\begin{abstract}
Rui Zhang, Qianqian Wu and Rutao Liu (iD *
Widespread exposure to ultrafine carbon black (UFCB) and its ability to cross the lung-blood barrier have raised concerns regarding its safety. Importantly, UFCB can bind with proteins in the biological fluid after entering a biological environment and immediately form a protein corona. The protein corona could govern its further fate in the biological environment. In this work, we investigated the effects of UFCB to the structure and activity of catalase (CAT) to explore its biocompatibility with CAT. FW $200(13 \mathrm{~nm})$ and tween 80 (T80) were used as the UFCB model and the dispersant. Electron microscopy and dynamic light scattering (DLS) were used to characterize the surface properties and size distribution of UFCB. Steady-state fluorescence combined with synchronous fluorescence and 3D fluorescence show that UFCB bound with CAT and formed the protein corona, resulting an exposure of the internal amino acids (mainly tryptophan and tyrosine) and a decrease of the hydrophobicity of the amino acids. The fluorescence lifetime measurement combined with UV-visible spectra measurement indicated that UFCB quenched the fluorescence of CAT statically and changed the framework of CAT. Circular dichroism (CD) spectra analysis indicated the increase of $\alpha$-helical content and the decrease of $\beta$-sheet structure in catalase, which in turn make the activity of CAT reduce as shown in the enzyme activity assay. The study demonstrated the negative effects of UFCB on proteins and stressed the urgency to conduct more investigation on the biosafety of its application.
\end{abstract}

\section{Introduction}

Over the past decade, nanotechnology has become an emerging field and consists of an extensive range of technologies processing materials that have at least one dimension between 1 and $100 \mathrm{~nm} \cdot{ }^{\mathbf{1 - 3}}$ Carbon-based nanoparticles such as ultrafine carbon black (UFCB) have been intentionally applied in producing inks, coatings, battery cathodes and rubber reinforcing agents. ${ }^{4,5}$ The properties of carbon nanoparticles differ significantly from those of bulk materials made of the same compound, making them exhibit distinct chemical and physical functional activities. ${ }^{6,7}$ Although the rapid increase in the development of carbon-based nanotechnology initiated considerable enthusiasm, the widespread use of nanomaterials, particularly carbon nanoparticles, has sparked intensive concerns in society due to their potential negative

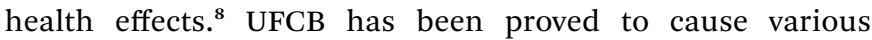

School of Environmental Science and Engineering, Shandong University, China-America CRC for Environment \& Health, 27\# Shanda South Road, Jinan 250100, Shandong Province, P. R. China. E-mail: rutaoliu@sdu.edu.cn; Fax: +86531-88364868; Tel: +86-531-88364868

$\dagger$ Electronic supplementary information (ESI) available: Dispersion effect of FW200 in tween 80, phase plot of the zeta-potential measurement, inner-filter effect of FW200/T80-CAT system, RLS spectra, homologous identity of bovine catalase and homo catalase. See DOI: 10.1039/c7ra03805d harmful effects. ${ }^{9,10}$ They are known to evoke pulmonary inflammation to pregnant mice. ${ }^{11}$ They stimulate both the elevation of macrophage and neutrophil number, and experiments with $\mathrm{CB}$ in mice have shown positive rates of lung tumors. ${ }^{12}$ Humans, especially workers employed in nanotechnology activities, can be exposed to UFCB through routes like oral instillation, inhalation, and the dermal route. Some of the UFCB particles may cross the epithelial pulmonary lining or stomach and thereafter reach systemic circulation. They can then cause effects to the organs and bind with enzymes in those organs.

The interaction of nanoparticles with proteins in biological fluids could be initiated after the nanoparticles invade the biological environment. They will form a dynamic structure called the protein-corona. ${ }^{\mathbf{1 3 , 1 4}}$ The composition of the external coating is determined by the size and surface properties of nanoparticles and equilibrium binding constants of each protein-corona. ${ }^{\mathbf{1 3 , 1 5}}$ The final corona (hard corona) is permanent and able to mediate their interaction with other parts of the cell. After reaching equilibrium, the particle will bind with the external protein and thereafter may interact with it. To date, most investigations on the protein-corona is about its biological effects on the cell recognition and internalization, ${ }^{\mathbf{1 3}}$ little focused on uncovering the interaction mechanism of nanoparticle and the protein. 
Binding on the nanoparticle surface can make proteins lose their native conformation. ${ }^{\mathbf{1 6}}$ Nanoparticles with different properties could cause changes to the secondary and tertiary structure of the protein in different degree. ${ }^{17}$ The alterations in the structure of the protein could then lead a loss of protein function. For instance, the adsorption of acetylcholinesterase (AChE) and butyrylcholinesterase (BChE) to carbon nanotubes could inhibit the activities of AChE and BChE; the external protein coat could make the reticuloendothelial system recognize nanomaterials and entrap them into the spleen and liver. ${ }^{13}$ Oxidative stress could be the most relevant pathogenetic pathway which links nanoparticle exposure to tissue damage. ${ }^{18}$ As an important antioxidant enzyme, catalase (CAT) plays a key role in protecting the cell from xenobiotics-induced stress tolerance. Moreover, numbers of studies confirmed that the nanomaterial invading could significantly change the CAT activity. ${ }^{\mathbf{1 9 , 2 0}}$ Nevertheless, the specific information of the interaction of CAT and nanoparticle is lacked.

Hence, the aim of our work was to test the conformational and functional changes of CAT caused by its binding with UFCB. Dynamic light scattering (DLS), transmission electron microscope (TEM) and scanning electron microscope (SEM) were used to characterize UFCB. UV-visible absorption, multiple spectra measurements, molecular docking and enzyme activity test were used to investigate the toxic effects of UFCB on the activity and conformation of CAT. The work unveiled the potential toxicity of UFCB to the most ubiquitous antioxidant enzyme and manifested the binding mode, binding parameters, binding sites and the conformational change of CAT through biophysical methods. Such explorations would aid in better comprehending the toxic mechanism of UFCB in vitro and bring about new scientific insights about other nanomaterials.

\section{Experimental}

\subsection{Materials and animals}

CAT was purchased from Sigma-Aldrich (St. Louis, Missouri, USA). $\mathrm{H}_{2} \mathrm{O}_{2}$ was purchased from Sinopharm Chemical Reagent Beijing Co., Ltd (Beijing, China). Tween 80 (T80), $\mathrm{NaH}_{2} \mathrm{PO}_{4}$ $\cdot 2 \mathrm{H}_{2} \mathrm{O}$ and $\mathrm{Na}_{2} \mathrm{HPO}_{4} \cdot 12 \mathrm{H}_{2} \mathrm{O}$ were purchased from Tianjin Damao Chemical Reagent Factory (Tianjin, China). $0.02 \mathrm{M}$ phosphate buffer $(\mathrm{pH}=7.4)$ was applied to control $\mathrm{pH}$.

\subsection{Particle preparation and characterization}

FW200 was heated at $200{ }^{\circ} \mathrm{C}$ for $120 \mathrm{~min}$ in an electric heater to eliminate endotoxins, then suspended by sonication in T80 solution $\left(\mathrm{C}_{\mathrm{T} 80}<1 \mu \mathrm{g} \mathrm{mL}^{-1}\right)$. The particle suspensions $\left(50 \mu \mathrm{g} \mathrm{mL}{ }^{-1}\right)$ were sonicated using a $250 \mathrm{~W}$ Scientz Sonifier SB-5200 DTD (Ningbo Scientz Biotechnology Co., Ltd, Ningbo, Zhejiang, China) for $10 \mathrm{~min}$ to mix and form a homogeneous dispersion. Particle suspensions were continuously cooled on ice during the sonication procedure, and then diluted as required before exposure procedure. The dispersions of FW200 in T80 solution were visualized with a transmission electron microscope (HRTEM; JEOL, Japan) and scanning electron microscope (SU8010, Hitachi, Japan). The zeta potential and hydrodynamic diameter of FW200 in T80 were characterized by dynamic light scattering (DLS) using a Malvern Zetasizer NanoZS (ZEN3600, Malvern, Worcestershire, UK) and the data were analyzed using the Dispersion Technology Software (DTS) version 5.0 (Malvern Instruments Ltd). DLS analysis of the suspension was performed on the raw UFCB suspension and the suspension after filtration through $0.8 \mu \mathrm{m}$ and $0.22 \mu \mathrm{m}$ syringe filters (Jin Long, Tianjin Branch billion Lung Experimental Equipment Co., Ltd; Tianjin, China).

\subsection{CAT activity assay}

The measurement of CAT activity was carried out according to the method of Hao et al. ${ }^{21}$ using $\mathrm{H}_{2} \mathrm{O}_{2}$ as the substrate. In brief, $2 \mu \mathrm{M}$ CAT was incubated with UFCB of various concentrations for $30 \mathrm{~min}$. Meanwhile, the concentration of UFCB was set in such a way that the CAT activity measured in its presence at time "zero" was not lower than $90 \%$ of that measured without UFCB. At appropriate time intervals $200 \mu \mathrm{L}$ aliquots of the incubated solution were added into $3 \mathrm{~mL} 10 \mathrm{mM} \mathrm{H}_{2} \mathrm{O}_{2}$. Then the decomposition rate of $\mathrm{H}_{2} \mathrm{O}_{2}$ was recorded by measuring the absorbance of $\mathrm{H}_{2} \mathrm{O}_{2}$ at $240 \mathrm{~nm} 30 \mathrm{~s}$ for over a period of $3 \mathrm{~min}$, which was defined as the CAT activity. Results were expressed as the relative activity of the blank control.

\subsection{Multiple spectra measurements}

All fluorescence spectra were recorded on a Spectrofluorimeter model F-4600 (Hitachi, Japan) in a $1.0 \mathrm{~cm}$ cuvette with a $150 \mathrm{~W}$ Xenon lamp on it. Fluorescence emission spectra were recorded over a wavelength range of 290-450 $\mathrm{nm}$ and the excitation wavelength was set at $280 \mathrm{~nm}$. The slit widths of emission and excitation were set at $5 \mathrm{~nm}$ and the scan speed was $1200 \mathrm{~nm} \min ^{-1}$. Photo multiplier tube voltage was $700 \mathrm{~V}$. Synchronous fluorescence spectra were recorded at the wavelength interval $(\Delta \lambda)$ of $15 \mathrm{~nm}$ and $60 \mathrm{~nm}$. The excitation wavelength scans ranged from 250 to $350 \mathrm{~nm}$. The threedimensional fluorescence measurements were conducted under the following conditions: the emission at $290-500 \mathrm{~nm}$, the excitation at $200-400 \mathrm{~nm}$.

FLS920 Combined Fluorescence Lifetime and Steady State Spectrometer (Edinburgh, U.K.) was used to record the timeresolved fluorescence spectra. The $\lambda_{\mathrm{em}}$ and $\lambda_{\mathrm{ex}}$ were set at 330 and $280 \mathrm{~nm}$, respectively.

UV-visible absorption spectra measurements were made on a UV-2450 spectrophotometer (Shimadzu, Kyoto, Japan) equipped with a $10 \mathrm{~mm}$ quartz cell and the emission wavelength was read at $190-450 \mathrm{~nm}$.

J-810 CD spectrometer (Jasco, Tokyo, Japan) was employed to record the CD spectra and the cell pathlength of $10 \mathrm{~mm}$ was used for the spectral range 190-260 $\mathrm{nm}$. The scanning speed was set at $200 \mathrm{~nm} \mathrm{~min}{ }^{-1}$. The CD signal was informed as the total signal (protein plus background) minus the background signal (FW200 plus PB). CDpro software (available at http:// lamar.colostate.edu/ sreeram/CDPro/) was employed to calculate ratios of secondary structures for the samples. 


\section{Results and discussion}

\subsection{Particle characterization}

As numerous works have reported the close relationship between the size of UFCB and its toxicity, we characterized FW200 by SEM, TEM and DLS to observe the particle morphology and evaluate the biological effects of FW200 in T80 solution. FW 200 UFCB was purchased from Evonik Degussa Corporation (Beijing, China). According to data sheet provided by the manufacture, the primary particle size of FW 200 is $13 \mathrm{~nm}$ and organic impurity content is less than 1\%. As a kind of amorphous carbon, the UFCB used in our work has a $350 \mathrm{~m}^{2} \mathrm{~g}^{-1}$ BET surface. ${ }^{22} \mathrm{FW} 200$ was dispersed in the T80 solution before use. SEM (Fig. 1A and B), TEM (Fig. 1C and D) and DLS (Fig. 2) were employed to characterize FW 200 suspension $\left(50 \mu \mathrm{g} \mathrm{mL}^{-1}\right)$ and obtain diameter, size distribution, photographs and morphology information about FW 200. The mean particle size measured in T80 was greater than that provided by the manufacture, suggesting that the particle tends to agglomerate in solution and form a polydisperse suspension. ${ }^{23}$ Fig. 1A shows that FW200 dispersed in T80 solution has a high aggregation level, which was in accordance with the results of DLS (Fig. 2). The corresponding image could be seen in Fig. S1.† The TEM demonstrated that FW 200 consisted of partially open to open chain - agglomerates (Fig. 1C). The agglomerate sizes of FW 200 ranged from $50 \mathrm{~nm}$ to more than $100 \mathrm{~nm}$ (Fig. 1B). High-resolution images (Fig. 1D) of FW 200 showed that the concentric layers with graphitic spacing of the UFCB resembled the nano-onions conformation.

DLS analysis conducted on three groups: the unfiltered (raw), the $0.8 \mu \mathrm{m}$ filtered and the $0.22 \mu \mathrm{m}$ filtered FW200

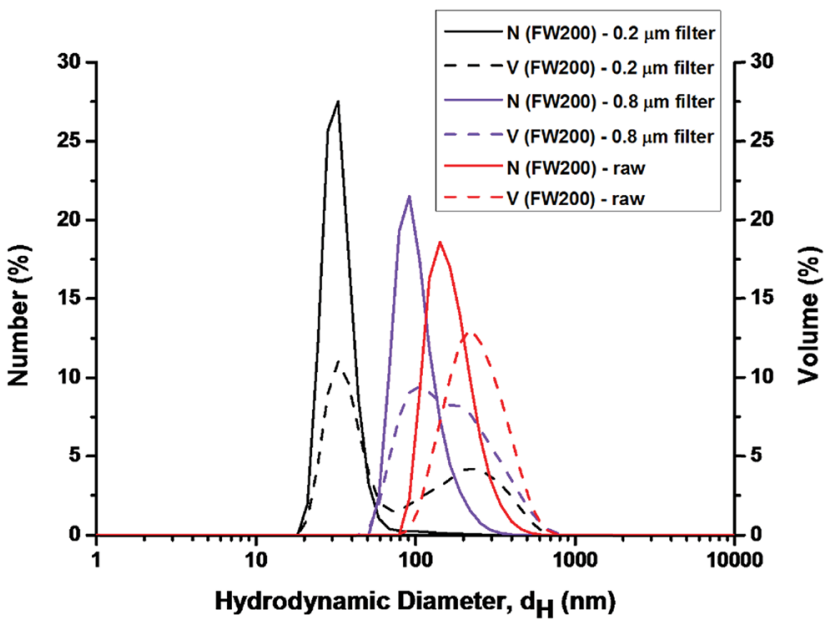

Fig. 2 Dynamic light scattering analysis of FW200 in T80 suspension, $C_{\mathrm{FW} 200}=50 \mu \mathrm{g} \mathrm{mL}^{-1}$.

suspension. The latter two groups were used to detect smaller particles that might exist in the FW200 suspension since larger particles can overshadow the smaller particles. ${ }^{24}$ In Fig. 2, the DLS analysis conducted on the raw FW200 system yielded a diameter of $180 \mathrm{~nm}$ for number-size-distribution and $220 \mathrm{~nm}$ for volume-size-distribution, which suggested that the particles in T80 solution agglomerated highly. In addition, the PDI (polydispersity index) and zeta-potential were 0.45 and $-3 \mathrm{mV}$ (corresponding phase plot was shown in Fig. S2 $\dagger$ ), an indication for a mildly polydisperse suspension. ${ }^{24}$ The suspension obtained from $0.8 \mu \mathrm{m}$ filter suggested the existence of particles with both number and volume peak sizes at about $100 \mathrm{~nm}$. After

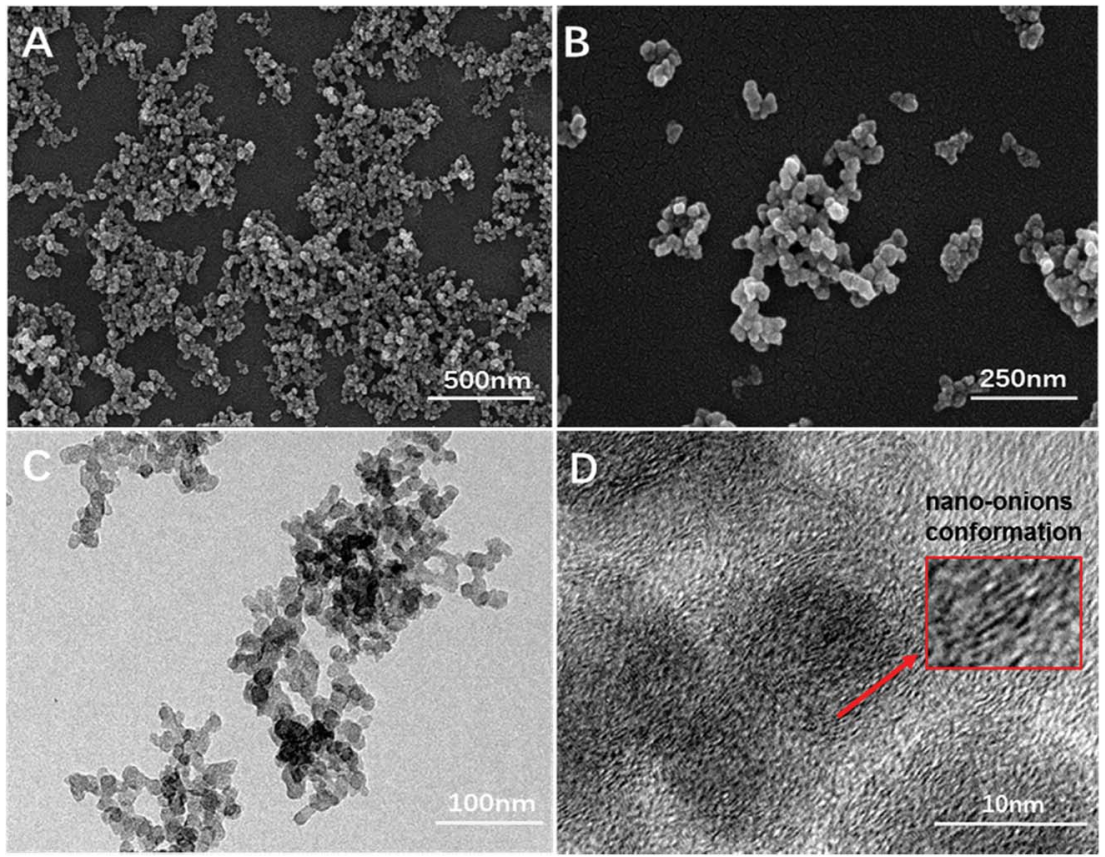

Fig. 1 Scanning electron microscope $(A$ and $B)$ and transmission electron microscope $(C$ and $D)$ images of FW 200 in T80 suspension, $C_{F W 200}=$ $50 \mu \mathrm{g} \mathrm{mL}^{-1}$. 

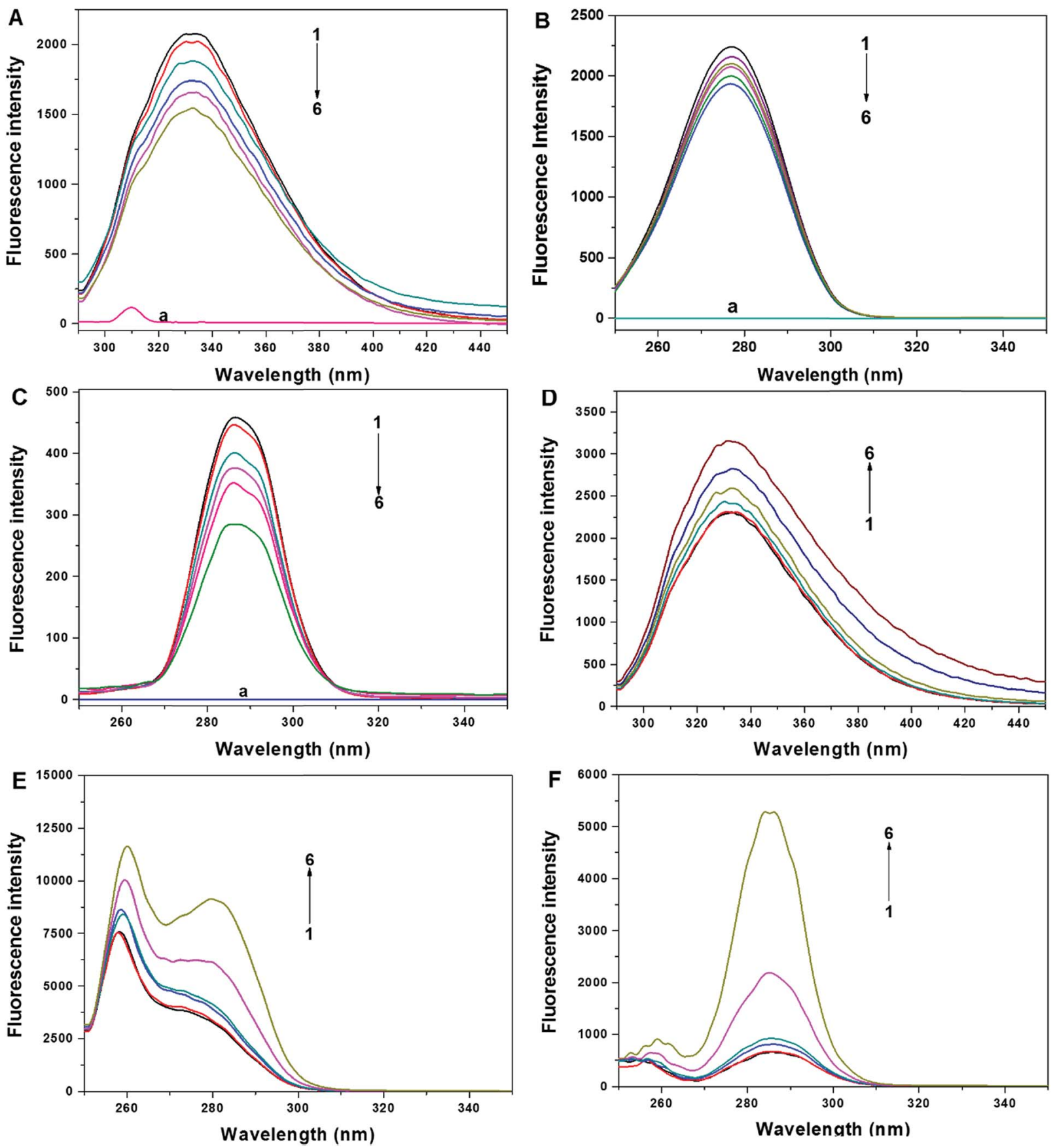

Fig. 3 CB-CAT system (A-C): steady-state fluorescence spectra: (A) synchronous fluorescence spectra $(\Delta \lambda=60 \mathrm{~nm})$ : (B) synchronous fluorescence spectra $(\Delta \lambda=15 \mathrm{~nm})$ : (C). T80-CAT system (D and E): fluorescence spectra: (D), synchronous fluorescence spectra ( $\Delta \lambda=60 \mathrm{~nm})$ : $(E)$ synchronous fluorescence spectra $(\Delta \lambda=15 \mathrm{~nm})$ : (F) conditions: $C_{\text {CAT }}: 2 \mu \mathrm{M}, C_{\mathrm{FW} 200}$ : 0, 10, 20, 30, 40, $50 \mu \mathrm{g} \mathrm{mL}^{-1}, C_{\mathrm{T} 80}: 0,1,5,10,20,30 \mu \mathrm{g} \mathrm{mL}$, $\mathrm{pH}=7.4, T=300 \mathrm{~K}$.

the filtration of $0.22 \mu \mathrm{m}$ filter, much smaller particles with number and volume peak sizes $(25 \mathrm{~nm})$ were detected in the filtrate. The PDI of suspensions obtained from $0.8 / 0.22 \mu \mathrm{m}$ filter was $0.37 / 0.35$, suggesting a more consistent system. DLS on unfiltered samples can only detect the largest particles in the suspension. Thus, the filtration of FW200 suspension was conducted to detect the smaller particles. The results of DLS confirmed the presence of nano-sized (less than $100 \mathrm{~nm}$ ) particles in the suspension.

\subsection{Steady-state fluorescence}

Protein has intrinsic fluorescence since it contains various chromophores, among which tryptophan (Trp), tyrosine (Tyr) and phenylalanine (Phe) account for the most quantum yield. ${ }^{25}$ The intrinsic fluorescence emission spectra has been widely used to investigate the structural transformation of proteins and nucleic acids. ${ }^{26,27}$ In this part, fluorescence and synchronous fluorescence spectra of FW200-CAT were measured to determine the effect of FW200 on the conformation of CAT. 

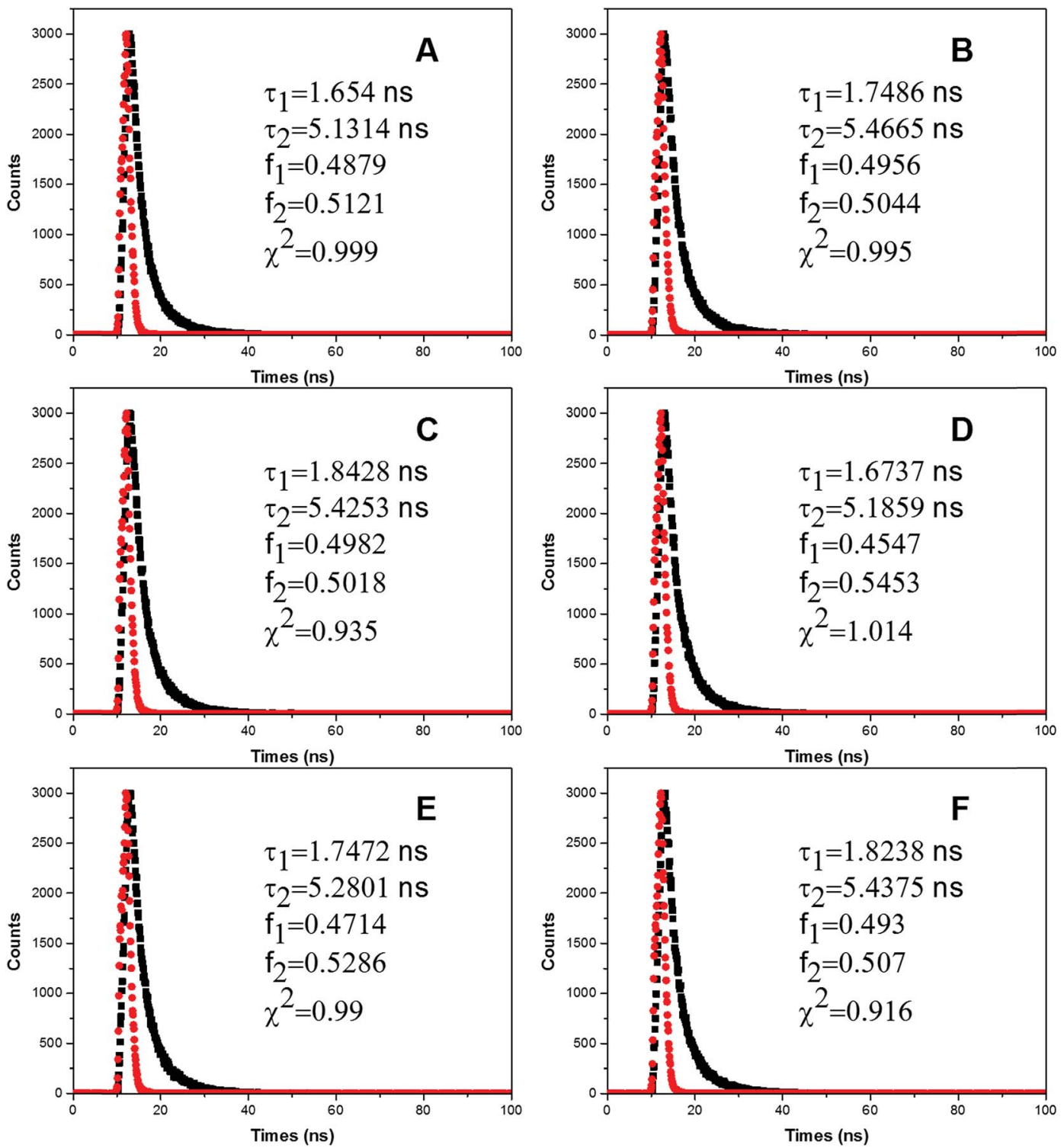

Fig. 4 Time-resolved fluorescence decay profiles of CAT.Conditions: $C_{\mathrm{CAT}}: 2 \mu \mathrm{M}, \mathrm{C}_{\mathrm{FW} 200}$ : $(\mathrm{A}-\mathrm{F}): 0,10,20,30,40,50 \mu \mathrm{g} \mathrm{mL}-1, \mathrm{pH}=7.4, T=$ $300 \mathrm{~K}, \lambda_{\mathrm{em}}=331 \mathrm{~nm}$.

Fig. 3A shows the fluorescence emission spectra of FW200-CAT system. As can be seen from it, the fluorescence intensity of CAT decreased gradually and the maximum fluorescence emission wavelength shifted from 330 to 334 with various concentrations of FW200 being titrated into CAT solution, which indicated that the conformation of CAT was changed by FW200 and the microenvironment of CAT tended to be hydrophilic.

Synchronous fluorescence reveals the characteristic information about tyrosine (Tyr) or tryptophan (Trp) residues, when the intervals $(\Delta \lambda)$ between excitation and emission wavelengths are set at 15 or $60 \mathrm{~nm},{ }^{28}$ respectively. Fig. $3 \mathrm{~B}(\Delta \lambda=60 \mathrm{~nm})$ and $\mathrm{C}$ $(\Delta \lambda=15 \mathrm{~nm})$ shows the synchronous fluorescence spectra of FW200-CAT system. As can be seen from it, the characteristic emission maximum of tryptophan and tyrosine decreased with slight red shift at the investigated concentration range. This suggests that the polarity around Tyr and Trp increased while the hydrophobicity of them reduced after the addition of FW200. Hydrophobic interaction which could affect the adsorption of protein to FW200 might be the main binding force between FW200 and CAT. ${ }^{17}$ To figure out the certain concentration of T80 under which the effect of T80 on the fluorescence of CAT could be neglected, the interaction of T80 and CAT was characterized by the fluorescence measurements. Fig. 3(D-F) shows the fluorescence emission spectra (D) and synchronous fluorescence spectra $(\mathrm{E}, \Delta \lambda=60 \mathrm{~nm}$ and $\mathrm{F}, \Delta \lambda=$ $15 \mathrm{~nm}$ ) of T80-CAT system, both the fluorescence and synchronous fluorescence intensity of CAT increased markedly with concentration of $\mathrm{T} 80$ ranging from 1 to $30 \mu \mathrm{g} \mathrm{mL} \mathrm{L}^{-1}$, but changes little when the concentration of T80 was less than or equal to $1 \mu \mathrm{g} \mathrm{mL} \mathrm{m}^{-1}$. In order to erase the disturbance of T80 to the FW200-CAT system, the concentration of T80 was set below $1 \mu \mathrm{g} \mathrm{mL}{ }^{-1}$. Furthermore, in order to detect the effect of pure 
Table 1 Fluorescence lifetime constants of the FW200-CAT systems at the following conditions: CAT: $2 \mu \mathrm{M}$; FW200 ( $\mu \mathrm{M}$ ): (a) 0, (b) 10, (c) 20, (d) 30 , (e) 40 , (f) $50 \mu \mathrm{g} \mathrm{mL}^{-1} ; \mathrm{pH}: 7.4 ; \mathrm{T}: 300 \mathrm{~K}$

\begin{tabular}{|c|c|c|c|c|c|c|c|}
\hline \multirow[b]{2}{*}{ System } & \multirow[b]{2}{*}{ Number } & \multicolumn{2}{|c|}{ Lifetime (ns) } & \multicolumn{2}{|c|}{ Amplitude (\%) } & \multirow[b]{2}{*}{$\tau_{\mathrm{AV}}$} & \multirow[b]{2}{*}{$\chi^{2}$} \\
\hline & & $\tau_{1}$ & $\tau_{2}$ & $A_{1}$ & $A_{2}$ & & \\
\hline \multirow[t]{6}{*}{ FW200-CAT } & a & 1.654 & 5.1314 & 0.4879 & 0.5121 & 3.4348 & 0.999 \\
\hline & $\mathrm{b}$ & 1.7486 & 5.4665 & 0.4956 & 0.5044 & 3.6239 & 0.995 \\
\hline & c & 1.8428 & 5.4253 & 0.4982 & 0.5018 & 3.6405 & 0.935 \\
\hline & d & 1.6737 & 5.1859 & 0.4547 & 0.5453 & 3.5889 & 1.014 \\
\hline & e & 1.7472 & 5.2801 & 0.4714 & 0.5286 & 3.6147 & 0.99 \\
\hline & f & 1.8238 & 5.4375 & 0.493 & 0.507 & 3.6559 & 0.916 \\
\hline
\end{tabular}

FW200 on the fluorescence and synchronous fluorescence spectra of the FW200-CAT system, we measured the fluorescence intensity of the pure FW200 solution (A-C, curve (a)), which indicated that pure FW200 did not exhibit any fluorescence and thus had no effect on both the fluorescence and synchronous fluorescence spectra of FW200-CAT system. The inner-filter effect that may exist in the FW200-CAT system was investigated and the results showed that it should not be neglected (Fig. S3†), so the inner-filter effect was eliminated in this experiment. ${ }^{29}$ The inner-effect of T80-CAT system was also measured and then eliminated in the same way and the absorption plot was shown in Fig. S4. $\dagger$

\subsection{Time-resolved fluorescence quenching of CAT in the presence of FW200}

Fluorescence quenching process occurred through three main mechanisms: dynamic quenching, static quenching or a combination of both. Dynamic quenching process happens when the quencher interacts with the excited state of the fluorophore and then decreases lifetime of the fluorophore. ${ }^{30}$ By contrast, the mechanism of static quenching refers to the quencher that directly quenches its original fluorescence by preventing the fluorophore from transforming into excited state. ${ }^{30}$ The most important fluorophore in the protein is tryptophan (Trp) residue, which shows multiple exponential decays and its rotamers play an important role in determining the fluorescence lifetime of the protein. ${ }^{31}$ In this paper, we have studied the influence of FW200 of various concentrations on the fluorescence lifetimes of CAT. T80 had little effect on the fluorescence lifetimes of CAT as its concentration was below $1 \mu \mathrm{g} \mathrm{mL} \mathrm{m}^{-1}$ (data not shown). Fig. 4 shows representative heterogeneous fluorescence decay of CAT with two lifetimes as the concentration of FW200 increases, this decay could be caused by the interconversion among the conformational substates in CAT. ${ }^{32}$ In Table 1, average fluorescence lifetimes were calculated to better interpret the effects of FW200 on CAT. The equation $\tau_{\mathrm{AV}}=\tau_{1}+\tau_{2}$ was employed to calculate the average lifetimes $\tau_{\mathrm{AV}}$, while $\tau_{1} / \tau_{2}$ and $\alpha 1 / \alpha 2(\alpha 1+\alpha 2=1)$ refer to the lifetime of the double exponential values and the proportions of $\tau 1 / \tau 2$, respectively.

From Table 1 it is readily seen that the fluorescence lifetime of CAT increased slightly, which indicates the conformation and the microenvironment of Tyr residues were affected. However, as the resonance light scattering (RLS) intensity of FW200-CAT system increased with the addition of FW200 (Fig. S5 $\dagger$ ), the lifetime increment of the system could also induced by the increasing RLS intensity. Though it is hard to directly identify the quenching mechanism of CAT via analyzing the lifetime of CAT, static quenching has a higher possibility to be the quenching mechanism of CAT. We will further investigate the quenching mechanism in the UV-vis absorption assay.

\subsection{UV-vis absorption measurement}

In order to investigate the backbone structure change of CAT and the quenching mechanism of its fluorescence, UV absorption spectra of CAT with and without the presence of FW200 was recorded and presented in Fig. 5A. As can be seen from Fig. 5A, an increase in absorbance at $213 \mathrm{~nm}, 280 \mathrm{~nm}$ and $406 \mathrm{~nm}$ was observed with the concentration of FW200 increasing from nil to $50 \mu \mathrm{g} \mathrm{mL}^{-1}$. The absorption at $213 \mathrm{~nm}, 280 \mathrm{~nm}$ and $406 \mathrm{~nm}$ reflect the information about the framework conformation of CAT, the $\pi \rightarrow \pi^{*}$ transition of the three aromatic amino acids (Trp, Tyr and Phe) and the characteristic absorbance of heme, respectively. ${ }^{33}$ The increase of the absorbance at $213 \mathrm{~nm}$ and
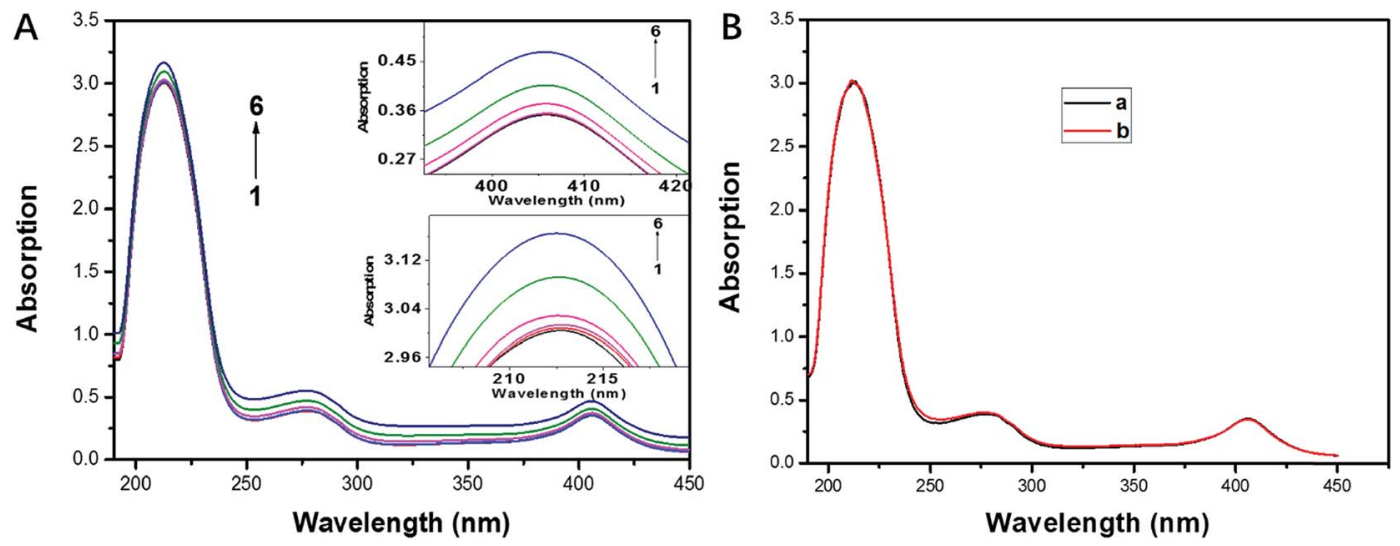

Fig. 5 (A): UV-visible absorption spectra of CAT. Conditions: (A): FW200-CAT, $C_{\mathrm{FW} 200}(1-6)=0,10,20,30,40,50 \mu \mathrm{gL}^{-1}, \mathrm{C}_{\mathrm{T} 80}<1 \mu \mathrm{g} \mathrm{mL}-1$ (B):

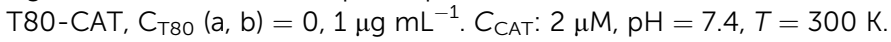


$280 \mathrm{~nm}$ indicates that FW200 interacted with CAT, changing the framework conformation of CAT and the microenvironment of the chromophores. Importantly, as the heme is an important component in constituting the active center of CAT, the increase of the absorbance at $406 \mathrm{~nm}$ suggests that FW200 bound close to the active center of CAT and might further affect the enzyme activity of CAT. Fig. 5B shows the UV absorption spectra of CAT-T80 system with the concentration of T80 was set at 0 and $1 \mu \mathrm{g} \mathrm{mL}{ }^{-1}$. As can be seen from Fig. 5B, T80 has little effect on the absorption spectra of CAT under $1 \mu \mathrm{g} \mathrm{mL}^{-1}$. In order to eliminate the effect caused by T80, we set the concentration of T80 below $1 \mu \mathrm{g} \mathrm{mL}{ }^{-1}$ in the measurement of the UV absorbance of FW200-CAT conjugate. UV-vis absorption spectra has been widely employed to figure out whether the protein and the ligand formed a ground state complex when they interacted with each other, which could be used to determine the quenching mechanism of the protein-ligand system. ${ }^{34}$ Static quenching can cause changes in absorption spectra of the protein resulting from complex formation at ground-state, by contrast, dynamic quenching could only affect the excited states of the fluorophores, and thus little change in the absorption spectra could be observed. The above results illustrated that a ground state complex of FW200-CAT was formed as the absorption spectra of CAT was changed and static quenching was proved to be the quenching mechanism of CAT. This verified the speculation made in the time-resolved fluorescence assay.

\subsection{D analysis}

As an ideal instrument to study structural changes in protein, three-dimensional fluorescence spectroscopy was employed to conduct non-intrusive measurements of protein-ligand interactions in this part. In order to further investigate the
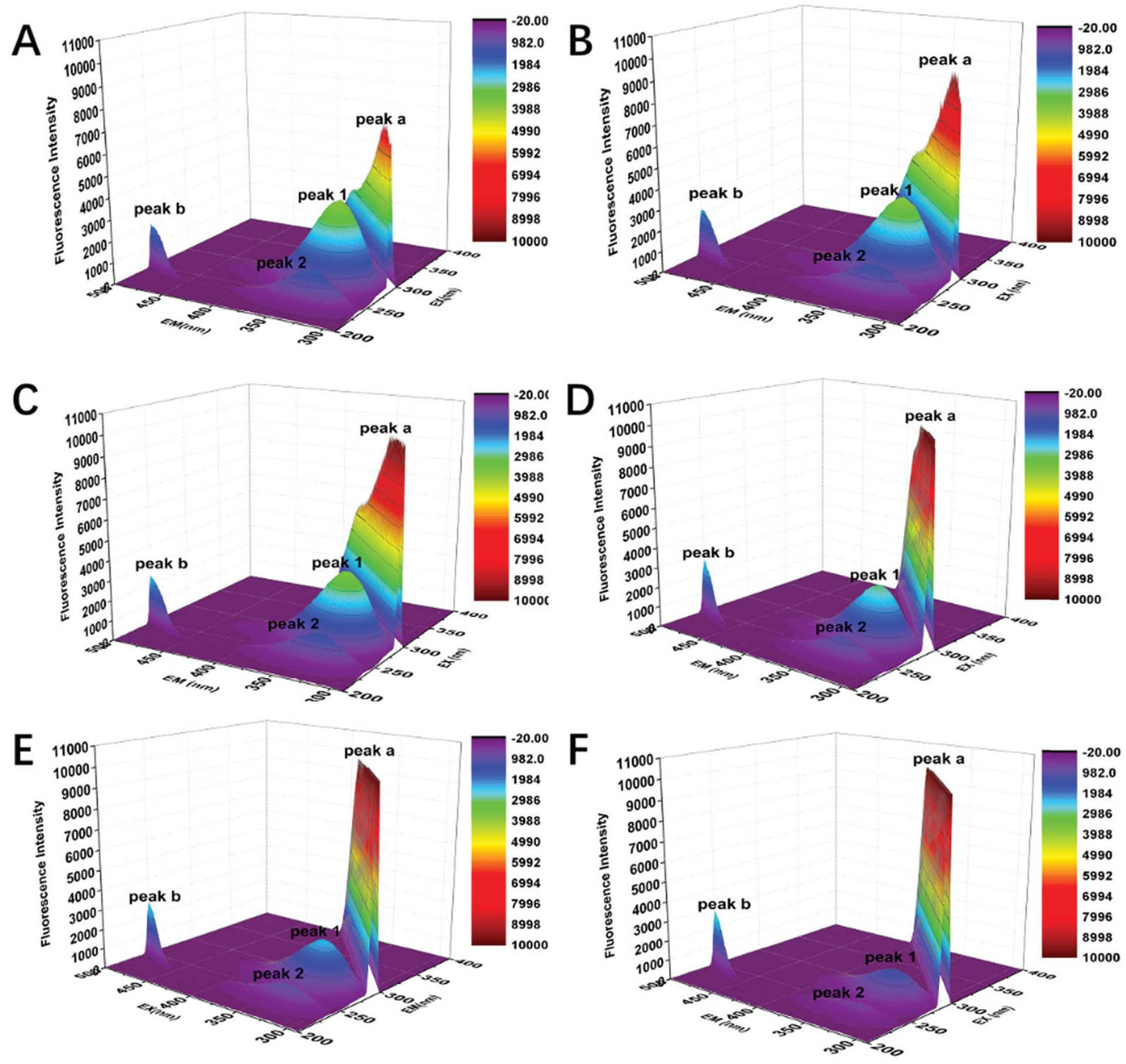

Fig. 6 Three-dimensional fluorescence spectra of CAT. Conditions: $C_{\text {CAT }}: 2 \mu \mathrm{M}, C_{\text {FW20o }}(A-F): 0,10,20,30,40,50 \mu g \mathrm{~mL}^{-1}, \mathrm{pH}=7.4, T=300 \mathrm{~K}$. 
Table 2 Three-dimensional characteristics of CAT and FW200-CAT complex

\begin{tabular}{|c|c|c|c|c|}
\hline \multirow[b]{2}{*}{ FW200 $\left(\mathrm{mg} \mathrm{L}^{-1}\right)$} & \multicolumn{2}{|l|}{ Peak (1) } & \multicolumn{2}{|l|}{ Peak (2) } \\
\hline & Position $\lambda_{\mathrm{ex}} / \lambda_{\mathrm{em}}\left(\mathrm{nm} \mathrm{nm}^{-1}\right)$ & Intensity $\mathrm{F}$ & Position $\lambda_{\mathrm{ex}} / \lambda_{\mathrm{em}}\left(\mathrm{nm} \mathrm{nm}^{-1}\right)$ & Intensity $\mathrm{F}$ \\
\hline 0 & $280 / 328$ & 4020 & $234 / 326$ & 1890 \\
\hline 0.001 & $280 / 330$ & 3875 & $234 / 334$ & 1546 \\
\hline 0.005 & $280 / 330$ & 3739 & $234 / 326$ & 1578 \\
\hline 0.025 & $280 / 330$ & 2993 & $234 / 330$ & 1285 \\
\hline 0.1 & $280 / 332$ & 2371 & $234 / 334$ & 979.5 \\
\hline 1 & $280 / 330$ & 1442 & $234 / 332$ & 627.4 \\
\hline
\end{tabular}

conformational changes of CAT caused by FW200, threedimensional fluorescence spectra of the FW200-CAT system was recorded. In Fig. 6 and Table 2, peak (a) and peak (b) are Rayleigh scattering peak $\left(\lambda_{\mathrm{ex}}=\lambda_{\mathrm{em}}\right)$ and second-order scattering peak $\left(\lambda_{\text {em }}=2 \lambda_{\text {ex }}\right)$, respectively. ${ }^{35}$ With the concentration of FW200 increasing, the intensity of peak (a) increased from 7055 to 10000 and that of peak (b) increased from 2040 to 2915, which revealed the fact that the protein-corona came into being after FW200 was added into CAT solution and the hydration radius of the protein-corona increased with the conformation of CAT being changed. Similar conclusion could be derived from the results of the RLS assay showed in Fig. S5. $\dagger$ Another two typical fluorescence peaks marked by peak (1) and peak (2) could be easily seen in the three-dimensional fluorescence spectra of FW200-CAT system. Peak (1) and peak (2) generated from the excitation of $280 \mathrm{~nm}$ and $234 \mathrm{~nm}$ and the emission wavelengths of these two peaks are both around $330 \mathrm{~nm}$. The shape and intensity of peak (1) revealed the spectral characteristic of Tyr and Trp residues and $\mathrm{n} \rightarrow \pi^{*}$ transition of polypeptide backbone structure $\mathrm{C}=\mathrm{O}$, since when CAT is excited at $280 \mathrm{~nm}$, it mostly reflects intrinsic fluorescence of Trp and Tyr residues. ${ }^{35}$ The quench of peak (1) indicates that the microenvironment and the conformation of CAT were changed. Peak (2) is another strong fluorescence peak and reveals the spectral characteristic of tryptophan and tyrosine residues as

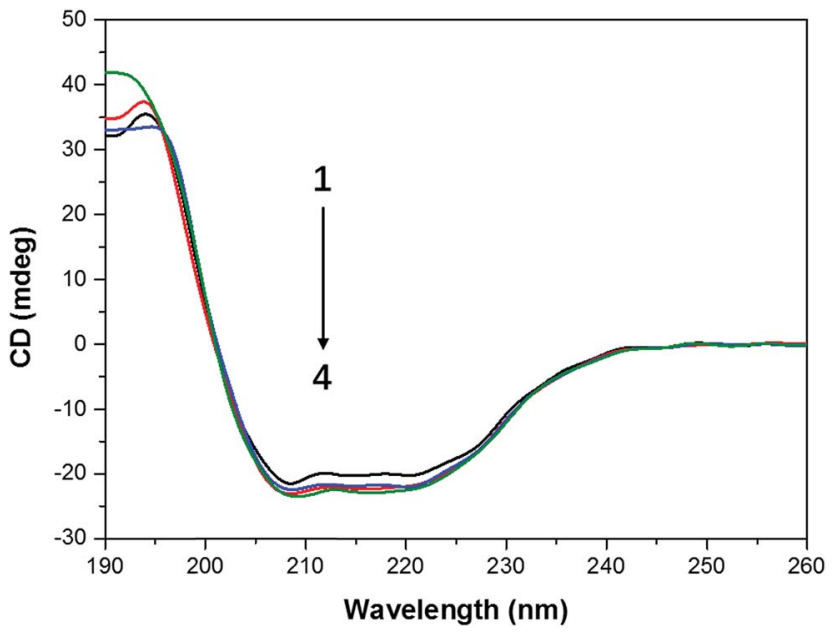

Fig. 7 CD spectra profile of FW200-CAT system. Conditions: $C_{\text {CAT }}: 250$ $\mu \mathrm{M}, \mathrm{C}_{\mathrm{FW} 200}: 0,10,30,50 \mu \mathrm{g} \mathrm{mL}-1, \mathrm{pH}=7.4, T=300 \mathrm{~K}$. well, but it simultaneously indicates the $\pi \rightarrow \pi^{*}$ transition of aromatic amino. ${ }^{35}$ The quench of these two peaks suggests that the interaction of CAT with FW200 led slight unfolding of the polypeptides of CAT and resulted in a change of the protein structure which play an important role in keeping the catalytic activity of the enzyme. ${ }^{36}$ This phenomenon confirmed the negative effects caused by FW200 to CAT and was in good accordance with the results of the fluorescence measurement and UV-vis experiment.

\subsection{Circular dichroism measurements}

When protein was bound with the ligand, the secondary structure of the protein can be changed by the ligand. ${ }^{37}$ Thus we conducted the circular dichroism measurements to figure out the changes in the secondary structure of CAT. The far ultraviolet CD spectra of CAT containing negative peaks at 209 and $222 \mathrm{~nm}$ reflects the characteristic of $\alpha$-helical structure (Fig. 7) and also indicate information on $\pi \rightarrow \pi^{*}$ transition and $n \rightarrow \pi$ transition, respectively. ${ }^{38} \mathrm{~T} 80$ has no effect on the secondary structure of CAT when its concentration was below $1 \mu \mathrm{g} \mathrm{mL}^{-1}$ (data not shown). In Table 3, it is interesting that FW200 caused a moderate change on the secondary structure of CAT with an $\alpha$ helical content increment $(\sim 6 \%)$ and a $\beta$-sheet content reduction $(\sim 6 \%)$. This phenomenon indicates that the hydrogenbonding networks of CAT were destroyed and the secondary structure was distorted.

\subsection{Effect of FW200 on activity of CAT}

According to above experiment results, the interaction of FW200 and CAT occurred and changed the conformation of

Table 3 The effects of FW200 on the secondary structure of CAT. Experimental conditions: $C_{\text {CAT }}: 2 \mu \mathrm{M}, C_{\mathrm{FW} 200}: 0,10,30,50 \mu \mathrm{gL}^{-1}, \mathrm{pH}$ $=7.4, T=300 \mathrm{~K}$

\begin{tabular}{lllll}
\hline & \multicolumn{3}{l}{ Secondary structural elements in CAT } \\
\cline { 2 - 5 } $\begin{array}{l}\text { Concentration OF FW200 } \\
\left(\mu \mathrm{g} \mathrm{mL}^{-1}\right)\end{array}$ & $\begin{array}{l}\alpha \text {-Helix } \\
(\%)\end{array}$ & $\begin{array}{l}\beta \text {-Sheet } \\
(\%)\end{array}$ & $\begin{array}{l}\beta \text {-Turn } \\
(\%)\end{array}$ & $\begin{array}{l}\text { Random } \\
(\%)\end{array}$ \\
\hline 0 & 47.3 & 17 & 12.8 & 23.9 \\
10 & 48.1 & 17 & 12.5 & 23 \\
30 & 49.1 & 16.7 & 12.9 & 23.5 \\
50 & 51.5 & 15.9 & 11.8 & 22.1
\end{tabular}




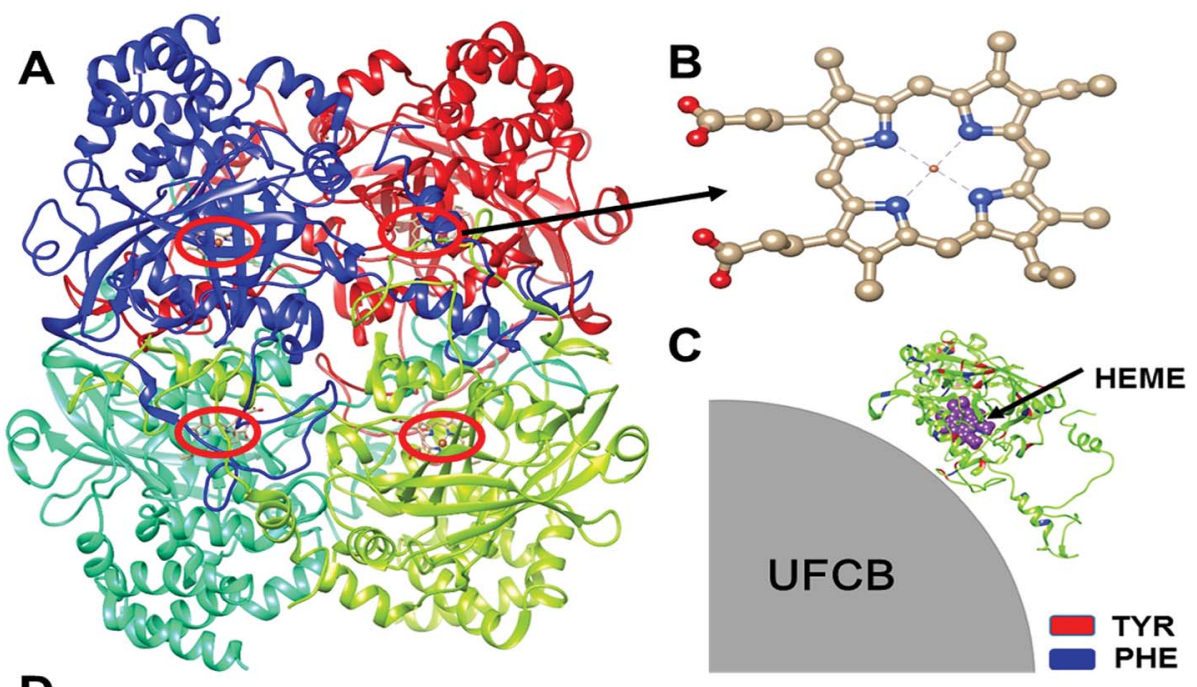

D

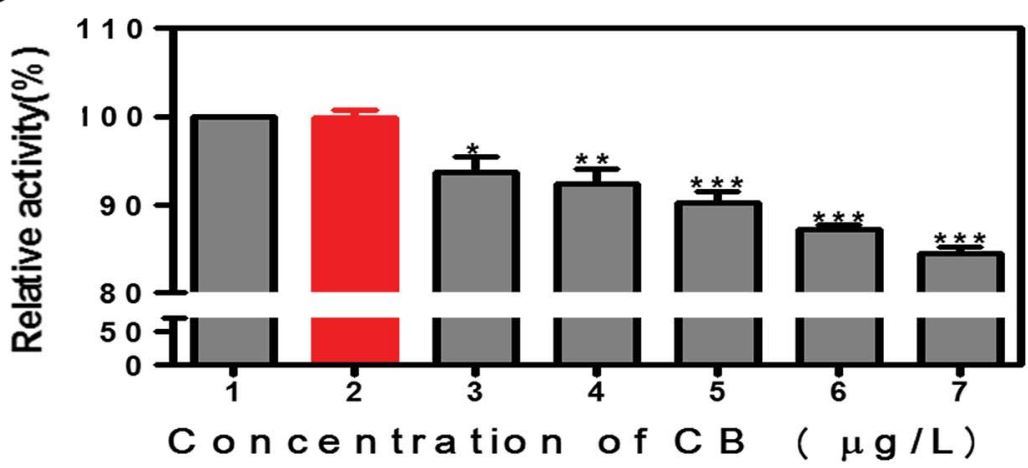

Fig. 8 Schematic diagram for the binding of the FW200 with CAT (A-C), (A) tetrameric structure of CAT; (B) the heme (active center) in CAT; (C) the enlarged view of FW200 close to the active site of CAT and the active site was shown in sphere mode and colored in purple, and the Tyr and

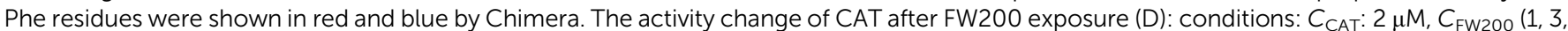
$4,5,6,7): 0,10,20,30,40,50 \mu \mathrm{gL}^{-1}, C_{\mathrm{T} 80}(2): 1 \mu \mathrm{g} \mathrm{mL}-1, \mathrm{pH}=7.4, T=300 \mathrm{~K}$.

CAT. In addition, it has been well known that the enzyme activity correlate with its conformation significantly. ${ }^{39}$ Therefore, the active assay is imperative to carry out with and without the presence of FW200 to clarify the relationship between structural change and activity variation of CAT. Bovine liver catalase was employed to perform this assay as it has a $91 \%$ homologous identity with the homo catalase (Fig. S6 $†$ ). Fig. 8A and B shows the tetrameric structure of CAT which includes four identical subunits. Each subunit possess a single heme which is the main part of CAT active center. The activity of CAT under various concentrations of FW200 was determined by setting the relative activity of CAT of the control group as $100 \%$. The effect of FW200 on CAT activity were presented in Fig. 8D, the catalase activity exhibited an obvious decrease compared to the control with the addition of FW200. As the concentration of FW200 increased from nil to $50 \mu \mathrm{g} \mathrm{mL}{ }^{-1}$, the activity of CAT decreased to $84 \%$ of the initial level. To determine the effects of T80 on the activity of CAT, the activity of CAT was explored after incubating with T80 $\left(1 \mu \mathrm{g} \mathrm{mL}{ }^{-1}\right)$. As shown by the red column, T80 caused little change on the CAT activity suggesting that the effect of T80 could be ignored in this assay. The conformational change of CAT derived from the above spectral analyses indicated that activity inhibition of CAT induced by FW200 could be partly ascribed to its structural changes. However, FW200 could also influence the activity of CAT through directly binding with the active center of CAT and the binding model of FW200 and CAT is shown in Fig. 8C. Similar results could be found in the work of Wei et al. who proved graphene oxide inhibited CAT activity through attacking the active center ${ }^{40}$ This hypothesis was proved by confirming the change of the microenvironment of Tyr343, Phe138 and Phe146 which bind near to the active center and have a close relationship with the activity of CAT.

\section{Conclusions}

The collective data presented herein demonstrate the toxicity of FW200 UFCB to CAT in vitro. We first characterized the morphology of FW200 in T80 solution, which showed that the size distribution of FW200 particles concentrated at $20 \mathrm{~nm}$, $100 \mathrm{~nm}$ and $200 \mathrm{~nm}$. This proved the nano-sized FW200 particles exist in T80 solution though the aggregation of the nanoparticle. The negative effects of FW200 to CAT on the molecular level was investigated by an array of techniques (spectra methods and enzyme activity assay), results showed that FW200 interacted with CAT and the binding site was close to the active center of CAT. The obviously quenched and red-shifted intrinsic 
fluorescence of aromatic amino acids in catalase indicate the decrease of microenvironment hydrophobicity. The interaction of FW200 and CAT decreased the $\alpha$-helix amount and increased the $\beta$-sheet fraction, leading to the mildly unfolding of the CAT conformation. The conformational changes of CAT further inhibited its enzymatic activity. Furthermore, the influence of UFCB on the CAT was concentration-dependent. Our work unveiled the toxicity of UFCB on the molecular level and highlighted the urgency for people to pay more attention to the toxicity of carbon-based nanoparticles.

\section{Conflicts of interest}

There are no conflicts of interest to declare.

\section{Acknowledgements}

This work is supported by NSFC (21277081, 21477067 and 21777088), the Cultivation Fund of the Key Scientific and Technical Innovation Project, Research Fund for the Doctoral Program of Higher Education and Ministry of Education of China $(708058,20130131110016)$ and independent innovation program of Jinan (201202083), Science and Technology Development Plan of Shandong Province (2014GSF117027) are also acknowledged.

\section{References}

1 E. Belade, L. Armand, L. Martinon, L. Kheuang, J. FleuryFeith, A. Baeza-Squiban, S. Lanone, M.-A. Billon-Galland, J.-C. Pairon and J. Boczkowski, Toxicol. in Vitro, 2012, 26, 57-66.

2 F. Schüth, Phys. Chem. Chem. Phys., 2011, 13, 2447-2448.

3 K. Shehzad, Y. Xu, C. Gao and X. Duan, Chem. Soc. Rev., 2016, 45, 5541-5588.

4 H. Kato, A. Nakamura, M. Horie, S. Endoh, K. Fujita, H. Iwahashi and S. Kinugasa, Carbon, 2011, 49, 3989-3997.

5 J. Zhang, M. Terrones, C. R. Park, R. Mukherjee, M. Monthioux, N. Koratkar, Y. S. Kim, R. Hurt, E. Frackowiak and T. Enoki, Carbon, 2016, 98, 8e732.

6 S. Lanone and J. Boczkowski, Curr. Mol. Med., 2006, 6, 651663.

7 G. Oberdörster, E. Oberdörster and J. Oberdörster, Environ. Health Perspect., 2005, 823-839.

8 J. Muller, F. Huaux and D. Lison, Carbon, 2006, 44, 10481056.

9 K. Shehzad, Y. Xu, C. Gao, H. Li, Z.-M. Dang, T. Hasan, J. Luo and X. Duan, ACS Appl. Mater. Interfaces, 2017, 9, 7591-7600.

10 M. Rahmat and P. Hubert, Compos. Sci. Technol., 2011, 72, 72-84.

11 P. Jackson, K. S. Hougaard, A. M. Z. Boisen, N. R. Jacobsen, K. A. Jensen, P. Møller, G. Brunborg, K. B. Gutzkow, O. Andersen and S. Loft, Nanotoxicology, 2012, 6, 486-500.

12 S. Yamamoto, M. Kakeyama, T. Kobayashi and H. Fujimaki, Toxicol. Appl. Pharmacol., 2005, 209, 51-61.

13 A. Pietroiusti, Nanoscale, 2012, 4, 1231-1247.
14 F. Ahmad, Y. Zhou, Z. Ling, Q. Xiang and X. Zhou, RSC Adv., 2016, 6, 35719-35730.

15 I. Lynch and K. A. Dawson, Nano today, 2008, 3, 40-47.

16 Z. Wu, B. Zhang and B. Yan, Int. J. Mol. Sci., 2009, 10, 41984209.

17 M. Sopotnik, A. Leonardi, I. Križaj, P. Dušak, D. Makovec, T. Mesarič, N. P. Ulrih, I. Junkar, K. Sepčić and D. Drobne, Carbon, 2015, 95, 560-572.

18 N. Li, T. Xia and A. E. Nel, Free Radical Biol. Med., 2008, 44, 1689-1699.

19 R. Klaper, J. Crago, J. Barr, D. Arndt, K. Setyowati and J. Chen, Environ. Pollut., 2009, 157, 1152-1156.

20 P. Prabhakar, U. A. Reddy, S. Singh, A. Balasubramanyam, M. Rahman, S. Indu Kumari, S. B. Agawane, U. Murty, P. Grover and M. Mahboob, J. Appl. Toxicol., 2012, 32, 436445.

21 F. Hao, M. Jing, X. Zhao and R. Liu, J. Photochem. Photobiol., $B, 2015,143,100-106$.

22 B. L'Azou, J. Jorly, D. On, E. Sellier, F. Moisan, J. Fleury-Feith, J. Cambar, P. Brochard and C. Ohayon-Courtès, Part. Fibre Toxicol., 2008, 5, 1.

23 M. Baalousha and J. R. Lead, Nat. Nanotechnol., 2013, 8, 308309.

24 C. Nickel, J. Angelstorf, R. Bienert, C. Burkart, S. Gabsch, S. Giebner, A. Haase, B. Hellack, H. Hollert and K. HundRinke, J. Nanopart. Res., 2014, 16, 2260.

25 X. Xie, Z. Wang, X. Zhou, X. Wang and X. Chen, J. Hazard. Mater., 2011, 192, 1291-1298.

26 B. K. Paul, D. Ray and N. Guchhait, Phys. Chem. Chem. Phys., 2013, 15, 1275-1287.

27 C. Jash, P. Basu, P. V. Payghan, N. Ghoshal and G. S. Kumar, Phys. Chem. Chem. Phys., 2015, 17, 16630-16645.

28 B. K. Paul, K. Bhattacharjee, S. Bose and N. Guchhait, Phys. Chem. Chem. Phys., 2012, 14, 15482-15493.

29 M. Van de Weert and L. Stella, J. Mol. Struct., 2011, 998, 144150.

30 S. E. Braslavsky, Pure Appl. Chem., 2007, 79, 293-465.

31 U. Anand, C. Jash, R. K. Boddepalli, A. Shrivastava and S. Mukherjee, J. Phys. Chem. B, 2011, 115, 6312-6320.

32 Z. Cao, R. Liu, Z. Dong, X. Yang and Y. Chen, Spectrochim. Acta, Part A, 2015, 136, 601-606.

33 Z. Cao, R. Liu and B. Yang, Spectrochim. Acta, Part A, 2013, 115, 457-463.

34 Y. Xiang and F. Wu, Spectrochim. Acta, Part A, 2010, 77, 430436.

35 R. Zhang, R. Liu and W. Zong, J. Agric. Food Chem., 2016, 64(34), 6630-6640.

36 E. Gökoğlu and E. Yılmaz, J. Fluoresc., 2014, 24, 1439-1445. 37 I. Matei and M. Hillebrand, J. Pharm. Biomed. Anal., 2010, 51, $768-773$.

38 X. Xie, W. Lü and X. Chen, J. Hazard. Mater., 2013, 248, 347354.

39 R. Lavery and S. Sacquin-Mora, J. Biosci., 2007, 32, 891-898. 40 X.-L. Wei and Z.-Q. Ge, Carbon, 2013, 60, 401-409. 